\title{
Fugl Meyer Assessment for Paralytic Glove with Micro Motor Configurations to Assist Post Stroke Therapy
}

\author{
Kalaiarasi Arumugam ${ }^{\text {a." }}$, Ashok Kumar Loganthan ${ }^{\mathrm{b}}$ \\ a Department of EEE, Sri Shakthi Institute of Engineering and Technology, Coimbatore- 641062, \\ Tamil Nadu, India. \\ ${ }^{\mathrm{b}}$ Department of EEE, PSG College of Technology, Coimbatore-641004, Tamil Nadu, India. \\ * Corresponding Author: akalaiarasieee@siet.ac.in
}

Received : $30^{\text {th }}$ July 2020, Accepted : $30^{\text {th }}$ August 2020

\begin{abstract}
Today, brain attack disorders are one of the most life threatening areas in the medical era, which the mankind is facing nowadays. Globally, more than 10,00,000people are subjected to brain attack disorders like hemiplegia and tremor, every year, where two thirds of them survive. Among the survival community, more than 80 per cent of them are subjected to long term impairment of their upper extremity. In order to treat the impairment, the survival group is subjected to medications and rehabilitation in order to improve their daily living. But the facilities are very limited in fast developing countries like India when compared to western standards. The rehabilitation given in corresponding with medications during the treatment period in hospitals does not give a complete recovery from disability. People from rural background could not meet their rehabilitation requirements even in the hospital during treatment and also when they are discharged to home after treatment from hospitals due to financial constraints and reachability. In order to motivate the survival group to fulfill their daily living and improve their lifestyle, this paper is focused on intelligent home based rehabilitation system at low cost, reliability, and affordability. One major movement disorder namely Upper Arm Hemiplegia was taken into account and visited few major hospitals around Coimbatore and Chennai for literature and case study. The facilities available in various hospitals and their drawbacks were analyzed. Based on the studies conducted at hospitals and taking advice from therapists, an innovative low cost home based rehabilitation device using Electro Mechanical systems has been developed to support samples who were used to impaired living even after treatments. To support Upper Arm Hemiplegia patients, the devices which were developed and experimented with Fugl Meyer Assessment to analyse the improvements in different functionalities are discussed in this paper.
\end{abstract}

Keywords: brain attack disorders, medical, hemiplegia, tremor, rehabilitation

\section{Introduction}

The issue of neurological deficits in young India has been of long interest to the neurologists in the country. The age group for brain disorders is varied from the age of 19-70 on different aspects which tends to have a unique set of causes and risk factors. Among the 
neurological deficits, Stroke and its side effects seek the immediate attention of medical experts. Stroke is one of the second leading causes of death and the major leading reason for disability worldwide [1]. The interruption of blood supply to the brain, which in turn blocking the blood flow or bursting the blood vessels is called stroke. The World Health Organization (WHO) states that "the rapid development of symptoms and signs of neurological interruption which lasts longer than $24 \mathrm{hrs}$ and in turn leading to death due to neurovascular issues” [2]. Globally, almost 25 million people are prone to stroke every year and 5\% lack survival [3]. Worldwide, the mortality rate of stroke is very much increasing and almost the results of stroke leads to functional impairment where more than $30 \%$ of people seek special intensive care and many prone to permanent disabilities [4] Stroke is characterized into two major pathophysiology's namely ischemic stroke and haemorrhagic stroke. The effectiveness of stroke depends on he impact of injury and its location. When a stoke is severe enough, it even leads to the sudden death of a person. The major symptom of stroke leads to dullness or weakness of the face, upper arm and lower arm or even any one side of the body. The other subordinate symptoms include speech difficulty, unconsciousness; dizziness etc [5]. Ischemic refers to sudden obstruction of blood supply to the brain which is almost accounted by $84 \%$ of the human crowd, whereas hemorrhagic stroke refers to sudden bleeding from any part of the brain which accounts for $16 \%$ of people globally [6]. On an average, the death rate in 30 days due to Ischemic stroke is $12 \%$, due to haemorrhagic stroke is $60 \%$. Among the above-said stroke cases, one- third of the stroke cases are preceded by a mini-stroke called Transient Ischemic Attacks (TIA), which interrupts a temporary flow of blood to the brain [7]. Accounting the above-mentioned stroke severities, the underdeveloped and the developing countries are subjected to stroke in a higher strike rate. Among them, nearly $85.5 \%$ of stroke deaths prevail and the numbers of disabilities are higher when compared with the developed countries [8].

\section{Epidemiology of Stroke}

The epidemiology of stroke would describe the distribution, prevalence and the incidences of stroke, in a global level and in India. The mortality and the morbidity estimates of stroke, globally and also in India are listed below in the below Table 1.

Table 1. Mortality and Morbidity Statistics

\begin{tabular}{|l|l|l|}
\hline & International Statistics & Indian Statistics \\
\hline Prevalence & $400-800 / 100000$ & $90-222 / 100000$ \\
\hline No of Death & 5.7 Million & 102,620 Million \\
\hline Acute Strokes per year & 16 Million & $1.44-1.64$ Million \\
\hline Fatality range & 28-30 day case from 17\%-35\% & 28-30 day case from18-41\% \\
\hline $\begin{array}{l}\text { Disability Adjusted Life Year } 28,500,000 \\
\text { (DALY) }\end{array}$ & $6,398,000$ \\
\hline
\end{tabular}

Source: Stroke in India Fact Sheet 


\section{International Scenario}

Over the past four decades, the standard incidences of stroke have shown a decremented figure by $20 \%$ of stroke deaths in developed countries, but the controversial increment of $36.7 \%$ is seen in middle class and underdeveloped countries [9]. The incidental and prevalence statistics of stroke in various regions of countries worldwide is shown in Table 2.

Table 2

\begin{tabular}{|l|l|l|l|}
\hline S. No. & Country & Incidental & Prevalence \\
\hline 1 & America & 0.9 Million & 4.8 Million \\
\hline 2 & Africa & 0.7 Million & 1.6 Million \\
\hline 3 & South East Asia & 1.8 Million & 4.5 Million \\
\hline 4 & Western Pacific & 3.3 Million & 9.1 Million \\
\hline 5 & Eastern Mediterranean & 0.4 Million & 1.1 Million \\
\hline 6 & Others & 9.0 Million & 30.7 Million \\
\hline
\end{tabular}

Source: Stroke in India Fact Sheet

\section{Indian Scenario}

According to the stroke registry database kept up by seven driving multi-specialty healing centers' in Coimbatore city, nearly 2000 samples reports of a stroke every year, of which 1500 pass on in clinics, 500 passes on at home and 10 to 20 samples pass on before reaching hospital facilities. PSG Hospitals announced that there were 1200 stroke cases around, on a normal of 120 cases each month. Correspondingly, Coimbatore Medical College Hospital (CMCH) detailed as many as 1500 cases, around of which 1050 were ischemic stroke cases and 450 were the hemorrhagic stroke. This year, they have received a normal of 35 stroke cases for each week, which demonstrates the number is practically going up. Samples originate from all age bunches however as of late they have gotten three samples a day from more youthful age group, where 10 percent stroke cases are seen among the age gathering of 16 to 24 years.

Table 3. Indian Scenario of Incidence and Prevalence of Stroke

\begin{tabular}{|l|l|l|l|}
\hline S. No. & Country & Incidental & Prevalence \\
\hline 1 & Mumbai & 0.2 Million & 1.4 Million \\
\hline 2 & Kolkata & 0.1 Million & 1.2 Million \\
\hline 3 & West Bengal & 1.1 Million & 1.5 Million \\
\hline 4 & Tamil Nadu & 1.3 Million & 1.6 Million \\
\hline
\end{tabular}

Source: Stroke in India Fact Sheet 
This neurological deficit not only affects the disabled but also their caregivers. Though many organized facilities prevail in many developed countries, the requirements are very scarce in India. The disability of the survival group necessitates the need of informal caregivers apart from their family members. In developed countries it is spent nearly $\$ 835$ per month [10]. In India, informal caregivers charge Rs.15,000 per month for occasional caregiving, which is highly alarming.

Since neurological disorders form a significant proportion in medical technology today, it quantifies the need for improved infrastructure, necessary resources for recovery and rehabilitation with affordable cost and care using man-machine interfacing techniques. Since neurological disorders and its side effects are of long-term disability, their physical effects are severe and affect the sensory systems, language, perception, and cognitive function. Rehabilitation for the survivors would help them to regain their lost movement skills, once it is treated in an effective way after the stabilization of the patient. But, the existing facilities of rehabilitation in India are restricted to limitations like less combination of movements, lack of tolerance limit, software issues and applicability.

\section{Design Considerations for Motor Actuated Rehabilitation System}

A motor is an electrical machine which converts direct form of electrical energy into mechanical energy. The objective of using a motor actuator is to design a handy or portable glove, driven by a low cost and low power motor. Since the actuation is both to and fro motion, the motor operating in both the directions for 0-180 degrees is needed to be chosen.

Assuming the mass of the arm to be actuated be $1 \mathrm{~kg}$. Therefore the force required to actuate the mass is $10 \mathrm{~N}$. The torque required to actuate the $10 \mathrm{~N}$, by the motor is required to be calculated.

$$
\text { Torque }=\text { Force }{ }^{*} \text { distance }
$$

For $\Theta=90$ degree and the assumed distance between the motor and the load be $2 \mathrm{~cm}$, $\mathrm{T}=10 * 2 * \sin (90)=20 \mathrm{Ncm}$

$$
\mathrm{T}=2 \mathrm{~kg}-\mathrm{cm}
$$

Therefore, a DC motors operating for the above torque with low power rating and 180 degree operation is $6 \mathrm{~V}$ micro DC motor. A non-automated glove, for finger kinematics using DC motor is represented as smart glove. The component requirements for the smart glove are discussed below in table 4. A micro DC motor is unlike a conventional motor which has selfsupporting skew wound copper winding instead of a copper winding.

A leather glove is a material which gives a high level of friction with grip over any object. A leather glove is fabricated with pores and grain which provides proper ventilation while use. These gloves provide a comfort feel when it is worn by samples for rehabilitation where the specifications are shown in table 5 . 
Table 4. Micro DC motor ratings

\begin{tabular}{|l|l|l|}
\hline S. No. & Parameter & Range \\
\hline 1 & Rated voltage & $6 \mathrm{~V}$ \\
\hline 2 & Current & $1.3 \mathrm{~A}$ \\
\hline 3 & Torque & $2.5 \mathrm{kgcm}$ \\
\hline 4 & Speed & $30 \mathrm{rpm}$ (no load) \\
\hline
\end{tabular}

Table 5. Glove specifications (Leather)

\begin{tabular}{|l|l|l|}
\hline S. No. & Specifications & Type \\
\hline 1 & Cuff & Knit wrist \\
\hline 2 & Glove cutt & Clute \\
\hline 3 & Length & $10-1 / 2$ ” \\
\hline 4 & Finger style & Full finger \\
\hline
\end{tabular}

A Regulated Power Supply (RPS) is an electronic circuit for DC voltage operations for a predetermined value at the load terminals irrespective of load fluctuations or variations. The circuit diagram of RPS is shown in Figure 1. A step down transformer is used to deduce the voltage limit from $230 \mathrm{~V}$ based on the application. The alternating voltage is converted into a pulsating DC voltage with the help of the bridge rectifier circuit. The resulting output voltage is given to the filter circuit for harmonic less operation.

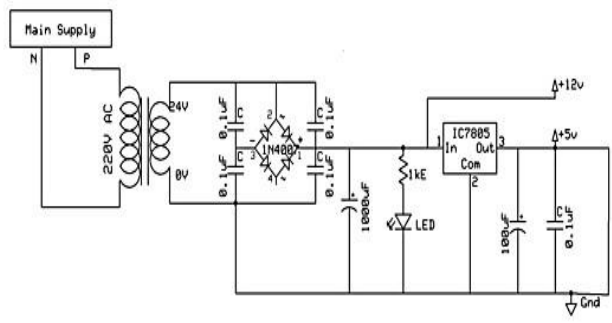

\section{Figure 1. Regulated Power Supply}

In the current industrial era, motor based actuations of medical devices are significant. Besides the availability of Pneumatic and Hydraulic systems, motor actuated systems possess the following advantages:

- High positioning performance

- Controlled flexibility

- No fluid leaks 
- High Energy efficiency

- Reliable

The motor actuated devices converts the rotational motion from the motor to a linear torque, which in turn creates the pull or pull force of the load by rotating the actuator.

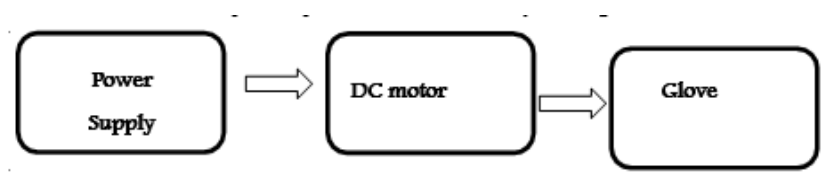

\section{Figure 2. General block diagram of smart glove for motor based rehabilitation}

The design as shown in figure 2 is aimed to meet the needs of independency, simplicity and affordability of stroke survivors. The design integrates a reflexive exercise machine with kinetic exercise. The smart glove is operated under single phase AC supply of $230 \mathrm{~V}$ being rectified to DC supplied to a $6 \mathrm{~V}, 0.5 \mathrm{~A}$ micro DC motor mounted on strap with a slide switch. The micro DC motor is connected to fingers using strong transparent wired strings. The transparent wired strings are tailored to a leather glove that can be worn by the patients. The operation of the switch enables the string wires to force the fingers to cause the flexion and extension.

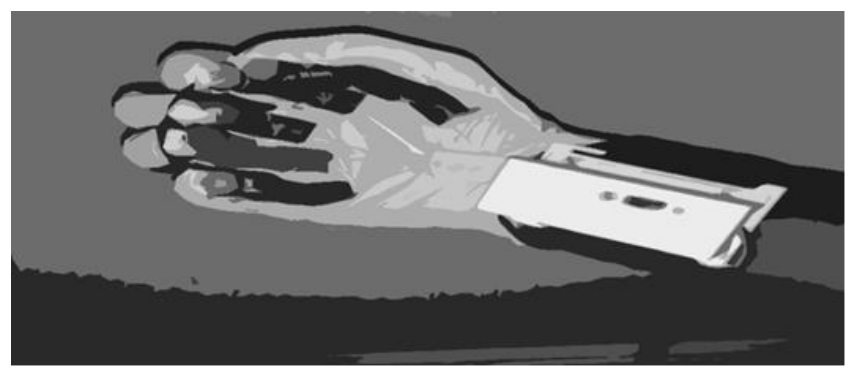

\section{Figure 3. Smart Glove for Motor based rehabilitation}

The smart glove shown in Figure 3 is operated under single phase AC supply of $230 \mathrm{~V}$ using an AC Adapter which is being rectified to DC supplied to a micro DC motor mounted on a wrist strap with a slide switch. The micro DC motor generates the required torque to actuate the fingers. The motor actuate the fingers using strong transparent wired strings to withstand any force given is tailored on a leather glove. A wrist strap is used to mount the DC motor for proper grip and support to the affected hand. The transparent wired strings are tailored to a leather glove which facilitates weightless movements can be worn by the samples in a flexible manner on the affected hand. The to and fro operation of the switch enables the transparent string wires to force the fingers to make the flexion and extension movements. 


\section{Results and Discussion}

The samples who were opted for experimental trial were assessed in prior to the therapy. The assessment criteria is categorized into three terms namely

Pre assessment (week 0, prior to the therapy)

Mid assessment (week 5, mid of the therapy)

Post assessment (week 10, after completion of the therapy)

The paresis level of the samples were analysed and the devices were chosen for the patient based on their severity and need of rehabilitation. The duration of the therapy is for about 30 minutes/day and subjected to extension based on the patient's interest and mental state.

The widely used method for assessment for upper arm and lower arm hemiplegia is Fugl Meyer Assessment [10]. The purpose of this assessment is to evaluate and measure the patient recovery of the survival group of upper arm hemiplegia. The major parameters of measurement using Fugl Meyer Assessment are Motor, Sensory, Balance, Range of Motion (RoM) and Joint pain.

All the electromechanical systems are applied to different samples and their assessment scores were generated with reference to the Fugl Meyer Assessment data sheet and the obtained values are converted into a Table form which is represented in section 5.3

Assuming that the variables of pre assessment to be ' $\mathrm{m}$ ' and the variables of post assessment to be ' $n$ ', the hypothesis testing is proposed in order to identify the significance of the developed product. Paired-t test is conducted to identify the mean difference among the population ' $m$ ' and 'n'. The paired sample t-test also referred as dependency t-test which is used to measure the significant improvement of the computed data. It is a factual system used to compute if the mean contrast between the two arrangements of the perception is zero. The paired t-test is conducted based on the following assumptions:

Null hypothesis: This hypothesis states that there exists no significant mean difference between the populations considered for paired t- test

Alternate hypothesis: This hypothesis states that there exists some notable mean difference between the populations considered for paired t- test. Here there exists upper tailed and lower tailed alternate hypotheses where the former denotes a positive mean difference while the later denotes a negative mean difference. 
Vol. 2 Iss. 2 Year 2020 Kalaiarasi Arumugam \& Ashok Kumar Loganthan /2020

Table 6. Fugl Meyer Assessment report for a patient using Motor Actuated Rehabilitation systems (Smart Glove)

\begin{tabular}{|c|c|c|c|c|c|c|}
\hline \multirow{2}{*}{\begin{tabular}{|l|} 
Parameter \\
Assessment
\end{tabular}} & \multicolumn{2}{|c|}{\begin{tabular}{|l|l|} 
Null & Partial \\
\end{tabular}} & \multirow[t]{2}{*}{ Full } & \multicolumn{3}{|c|}{ Assessment } \\
\hline & \multicolumn{2}{|c|}{ Scores } & & Pre & Mid & Post \\
\hline \multicolumn{7}{|l|}{ Upper Extremity } \\
\hline Flexors & 0 & 1 & 2 & 0 & 0 & 0 \\
\hline Extensors & 0 & 1 & 2 & 0 & 1 & 0 \\
\hline I. Reflex activity & 0 & 2 & 4 & 1 & 1 & 0 \\
\hline Shoulder retraction & 0 & 1 & 2 & 1 & 1 & 2 \\
\hline Shoulder elevation & 0 & 1 & 2 & 1 & 1 & 1 \\
\hline Shoulder abduction & 0 & 1 & 2 & 1 & 1 & 1 \\
\hline Shoulder external rotation & 0 & 1 & 2 & 1 & 1 & 1 \\
\hline Elbow flexion & 0 & 1 & 2 & 0 & 0 & 0 \\
\hline Forearm supination & 0 & 1 & 2 & 1 & 1 & 1 \\
\hline Shoulder adduction & 0 & 1 & 2 & 1 & 1 & 2 \\
\hline Elbow extension & 0 & 1 & 2 & 1 & 1 & 2 \\
\hline Forearm pronation & 0 & 1 & 2 & 1 & 1 & 1 \\
\hline II. Volatile motion within synergies & 0 & 9 & 18 & 8 & 8 & 11 \\
\hline Hand to lumbar spine & 0 & 1 & 2 & 0 & 0 & 0 \\
\hline Shoulder flexion & 0 & 1 & 2 & 1 & 1 & 1 \\
\hline Pronation-supination & 0 & 1 & 2 & 0 & 0 & 0 \\
\hline III. Volitional motion mixing synergies & 0 & 3 & 6 & 1 & 1 & 1 \\
\hline Shoulder abduction & 0 & 1 & 2 & 1 & 1 & 1 \\
\hline Shoulder flexion & 0 & 1 & 2 & 1 & 1 & 2 \\
\hline Pronation-supination & 0 & 1 & 2 & 0 & 1 & 2 \\
\hline IV. Volitional motion with no synergies & 0 & 3 & 6 & 2 & 3 & 5 \\
\hline V. Normal reflex activity & 0 & 1 & 2 & 1 & 1 & 2 \\
\hline TOTAL A (Upper Extremity) & 0 & 18 & 36 & 13 & 14 & 19 \\
\hline \multicolumn{7}{|l|}{ B. Wrist } \\
\hline Stability at $15 \%$ dorsiflexion & 0 & 1 & 2 & 0 & 1 & 1 \\
\hline Repeated dorsiflexion & 0 & 1 & 2 & 0 & 2 & 2 \\
\hline Stability at $15 \%$ dorsiflexion & 0 & 1 & 2 & 1 & 1 & 2 \\
\hline Repeated dorsiflexion & 0 & 1 & 2 & 1 & 1 & 2 \\
\hline Circumduction & 0 & 1 & 2 & 1 & 1 & 2 \\
\hline B. Wrist & 0 & 5 & 10 & 3 & 6 & 9 \\
\hline \multicolumn{7}{|l|}{ C. Hand } \\
\hline Mass flexion & 0 & 1 & 2 & 0 & 1 & 2 \\
\hline Mass extension & 0 & 1 & 2 & 1 & 2 & 2 \\
\hline Flexion in PIP and DIP & 0 & 1 & 2 & 0 & 1 & 1 \\
\hline Thumb adduction & 0 & 1 & 2 & 0 & 1 & 2 \\
\hline Opposition & 0 & 1 & 2 & 1 & 1 & 2 \\
\hline Cylinder grip & 0 & 1 & 2 & 1 & 2 & 2 \\
\hline Spherical grip & 0 & 1 & 2 & 1 & 1 & 1 \\
\hline C. Hand & 0 & 7 & 14 & 4 & 8 & 10 \\
\hline
\end{tabular}


Vol. 2 Iss. 2 Year 2020 Kalaiarasi Arumugam \& Ashok Kumar Loganthan/2020

\begin{tabular}{|l|l|l|l|l|l|l|}
\hline D. Coordination speed & 0 & 1 & 2 & 1 & 1 & 1 \\
\hline Tremor & 0 & 1 & 2 & 1 & 1 & 2 \\
\hline Dysmetria & 0 & 1 & 2 & 1 & 1 & 2 \\
\hline Time & 0 & 3 & 6 & 3 & 3 & 4 \\
\hline D. Coordination speed & 0 & 33 & 66 & 23 & 32 & 45 \\
\hline Total A D & & & & & & \\
\hline H. Sensation & 0 & 1 & 2 & 0 & 1 & 2 \\
\hline Light touch upprarm,fore arm & 0 & 1 & 2 & 0 & 1 & 1 \\
\hline Light touch palmer surface of hand & 0 & 1 & 2 & 0 & 0 & 1 \\
\hline Position Shoulder & 0 & 1 & 2 & 1 & 1 & 2 \\
\hline Position Elbow & 0 & 1 & 2 & 0 & 0 & 0 \\
\hline Position Wrist & 0 & 1 & 2 & 0 & 1 & 2 \\
\hline Position thumb & 0 & 6 & 12 & 1 & 4 & 8 \\
\hline H. Sensation & & & & & & \\
\hline I. Passive Joint Motion & 0 & 4 & 8 & 3 & 3 & 4 \\
\hline Shoulder & 0 & 2 & 4 & 2 & 3 & 4 \\
\hline Elbow & 0 & 2 & 4 & 1 & 2 & 3 \\
\hline Forearm & 0 & 2 & 4 & 2 & 3 & 3 \\
\hline Wrist & 0 & 2 & 4 & 2 & 2 & 2 \\
\hline Fingers & 0 & 12 & 24 & 10 & 13 & 16 \\
\hline I. Passive Joint Motion & & & & & & \\
\hline J. Joint Pain & 0 & 4 & 8 & 3 & 3 & 3 \\
\hline Shoulder & 0 & 2 & 4 & 2 & 2 & 3 \\
\hline Elbow & 0 & 2 & 4 & 2 & 3 & 4 \\
\hline Forearm & 0 & 2 & 4 & 2 & 3 & 3 \\
\hline Wrist & 0 & 2 & 4 & 2 & 3 & 3 \\
\hline Fingers & 0 & 12 & 24 & 11 & 14 & 17 \\
\hline I. Joint Pain & & & \\
\hline
\end{tabular}

The consolidated scores obtained during pre, mid and post phases of assessment were consolidated in the Table 6 .

Table 7

\begin{tabular}{|l|l|l|l|}
\hline Parameter & Pre-assessment & Mid-assessment & Post-assessment \\
\hline A. Upper & 13 & 17 & 20 \\
\hline B. Wrist & 3 & 6 & 9 \\
\hline C. Hand & 4 & 9 & 12 \\
\hline D. & 3 & 3 & 4 \\
\hline Total A-D & 23 & 35 & 45 \\
\hline Sensation & 1 & 4 & 8 \\
\hline Joint pain & 10 & 13 & 16 \\
\hline Passive Joint 11 & 14 & 17 \\
\hline
\end{tabular}


Vol. 2 Iss. 2 Year 2020 Kalaiarasi Arumugam \& Ashok Kumar Loganthan /2020
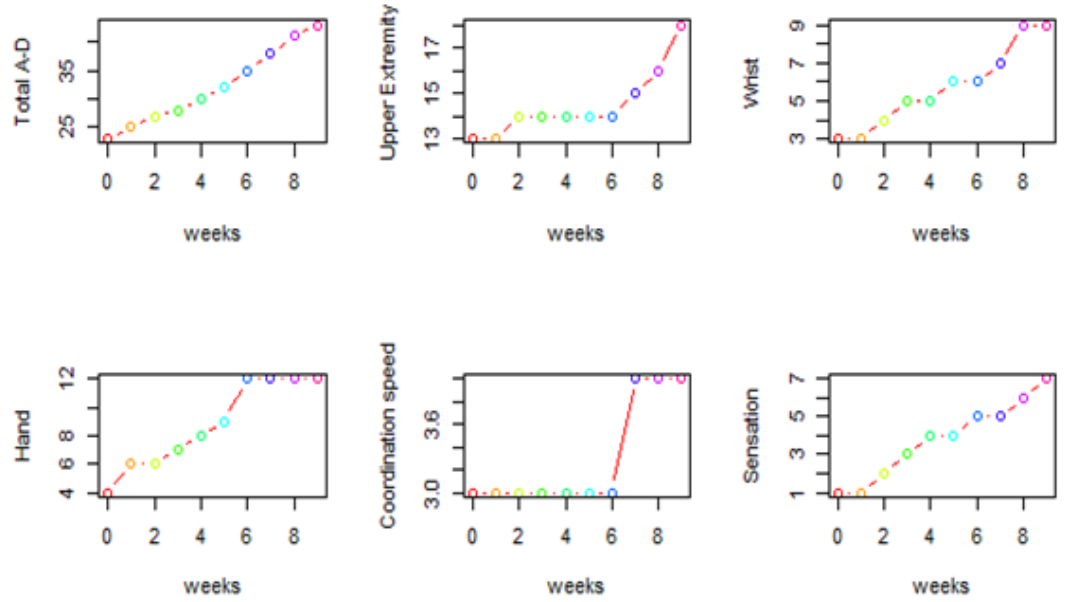

Figure 4. Scatter plot for Motor Actuated Rehabilitation systems (Smart Glove)

The scatter plot shown in Figure 4 infers how the major parameters of Fugl Meyer Assessment are affected over the period of assessment. The $\mathrm{x}$-axis predictor variable represents the number of weeks of assessment and the y-axis response variable represents the motion parameters of Fugl Meyer Assessment. The linear scatter plot obtained across the weeks demonstrates a linear model of data with no outliers.

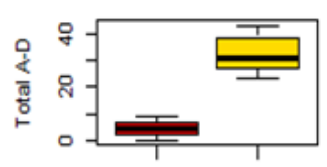

weeks

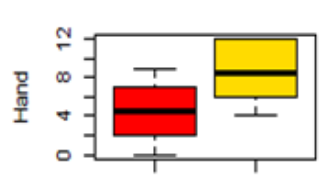

weeks

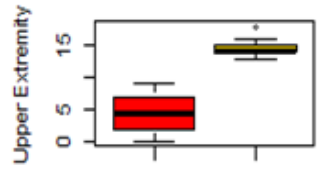

weeks

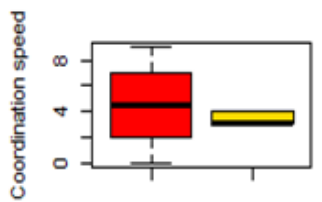

weeks

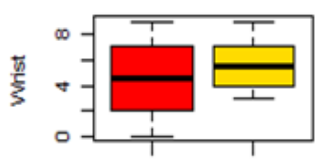

weeks

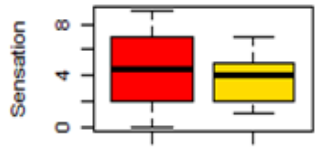

weeks

\section{Figure 5. Box plot for Motor Actuated Rehabilitation system (Smart Glove)}

The corresponding boxplot shown in Figure 5also proves the evidence for a normalized, clean data with no outlier extremes. The factors of Sensation, coordination speed, Hand, Upper Extremity, Wrist proves to vary normally across the weeks and hence the predictor and response variables prove to be in a linear modelling. Thus, the plots ensure reasonable linear improvements over the scale. 
Vol. 2 Iss. 2 Year 2020 Kalaiarasi Arumugam \& Ashok Kumar Loganthan/2020

Table 7 Statistical analysis results

\begin{tabular}{|l|l|l|l|l|l|l|}
\hline S. No. & Pre & Post & t value & P value & DoF & Mean Diff \\
\hline 1 & 22 & 43 & 1.9800 & 0.116 & 5 & 4.438 \\
\hline 2 & 23 & 45 & 1.9363 & 0.088 & 5 & 4.571 \\
\hline 3 & 21 & 42 & 2.0276 & 0.088 & 5 & 4.285 \\
\hline 4 & 17 & 35 & 1.7842 & 0.124 & 5 & 4.571 \\
\hline 5 & 18 & 37 & 1.8332 & 0.088 & 5 & 4.857 \\
\hline
\end{tabular}

From the Table 7, it is observed that, the difference between the pre assessment status at week 0 and the post assessment status at week 10 shows a reasonable improvement in the Kinematics of motion of the samples subjected to trial. Also, the obtained t-values from all the five experimental trials seem to be positive. The p-values have confirmed that there exists a mean difference between the two vectors ' $m$ ' and ' $n$ ' since they are greater than 0.05 . The mean difference is said to be positive from the observed positive't' values.

Even in this case of the assessment performed by therapist, the datasets favor alternate hypothesis that signifies there is a change in the assessment made across the weeks. This positive change thus drives the functionality of the product in improvising the samples motion control. Thus according to the various assessments done with the help of $\mathrm{R}$ tool the stability of the product is recognized. Hence the product proves to be helpful in bringing about the movements of the upper arm of the patient thus bringing about noteworthy changes in the lifestyle of the stroke survival group.

\section{Conclusion and Future work}

With reference to the proposed design concepts and operational benefits of various rehabilitation systems using electromechanical systems provided for Upper Arm Hemiplegia and tremor victims were briefed. The experimental trials have been induced on the victims subjected to home-based rehabilitation and the assessment results have been obtained using the Fugl Meyer Assessment using ANOVA analysis using the $\mathrm{R}$ - tool for upper arm hemiplegia results have been measured using an oscilloscope. To summarize, the design concepts proposed in the objectives proves to be a wearable device using electromechanical concepts. The results obtained from the experimental trials can be contributed to the fact that the rehabilitation devices can be used for home-based rehabilitation. The viability of the devices has shown that it is feasible to be implemented in practice through repetitive activities. This design may not only favor the victims of stroke, but it also adds value to the consistent physiotherapists or medical experts. The benefits of these proposed devices are easily accessible, reduced time management, and the cheaper cost which would ensure the effectiveness to be used as a product. Beyond home based rehabilitation, the medical practitioners can use these projects at their clinics and recommend the usage of these devices. Also, by extending the time of operation of the devices could also bring more enhancements in the life of the patients. 
The traditional therapies required for paralysis treatment are enormous in the medical instrumentation field. Besides the explosion in new rehabilitation systems available in the global market for paralysis, the therapies available for lower arm hemiplegia are very limited in developing countries. Compared to upper arm hemiplegia, the facilities available for lower arm hemiplegia are very limited and complicated. Therefore, reliable and cheaper devices based on electromechanical systems similar to the proposed concepts in this thesis can be designed, where the stroke victims with lower extremity will be highly motivated to achieve their locomotive targets in daily living. Also to improve the daily monitoring of the samples affected by paralysis, methodologies to collect data remotely can be implemented. The dataset may be used for further analysis based on the requirement. Wireless operations can be implemented to enable remote operations. Medical experts could be able to monitor the results obtained, remotely and thus favouring instant result analysis.

\section{References}

[1] S. J. Page, S. E. Wallace, Speech language pathologists opinions of constraint-induced language therapy, Topics in stroke rehabilitation, 21 (2014) 332-338.

[2] WHO, Preventing Chronic Diseases: A vital investment,Geneva, Switzerland. 2016.

[3] A. C. Recio, D. Becker, M. Morgan, N. R. Saunders, L. P. Schramm, J. W. McDonald, Use of a virtual reality physical ride-on sailing simulator as a rehabilitation tool for recreational sports and community reintegration: a pilot study, American journal of physical medicine \& rehabilitation, 92 (2013)1104-1109.

[4] B S. N.ailey, E. C. Hardin, R. Kobetic, L. M. Boggs, G. Pinault, R. J. Triolo, Neurotherapeutic and neuroprosthetic effects of implanted functional electrical stimulation for ambulation after incomplete spinal cord injury, Journal of Rehabilitation Research \& Development, 47 (2010) 7-16.

[5] O. Baus, S. Bouchard, Moving from virtual reality exposure-based therapy to augmented reality exposure-based therapy: a review, Frontiers in human neuroscience, 8 (2014) 112.

[6] W. Chen, F. Hopfner, J. S. Becktepe, G. Deuschl, Rest tremor revisited: Parkinson's disease and other disorders, Translational neurodegeneration, 6 (2017) 1-8.

[7] S. Choi, K. Jung, S. D. Noh, Virtual reality applications in manufacturing industries: Past research, present findings, and future directions, Concurrent Engineering, 23 (2015) 40-63.

[8] E. Christman, K. McAllister, K. Claar, S. Kaufman, S. J. Page, Occupational therapists' opinions of two pediatric constraint-induced movement therapy protocols, American Journal of Occupational Therapy, 69 (2015) 1-7. 
Vol. 2 Iss. 2 Year 2020 Kalaiarasi Arumugam \& Ashok Kumar Loganthan /2020

[9] L. Daniel, W. Howard, D. Braun, S. J. Page, Opinions of constraint-induced movement therapy among therapists in southwestern Ohio, Topics in stroke rehabilitation, 19 (2012) 68-275.

[10] D. Novak, J. Ziherl, A. Olenšek, M. Milavec, J. Podobnik, M. Mihelj, M. Munih, Psychophysiological responses to robotic rehabilitation tasks in stroke, IEEE Transactions on neural systems and rehabilitation engineering, 18 (2010) 351-361.

\section{Acknowledgements: NIL}

Conflict of interest: NIL

About the License: (C) 2020 The Authors. This work is licensed under a Creative Commons Attribution 4.0 International License which permits unrestricted use, provided the original author and source are credited. 